\title{
Transient membrane potential after concentration step: a new method for advanced characterization of ion- exchange membranes
}

\author{
Marc Fernández de Labastida ${ }^{1,2}$, Andriy Yaroshchuk ${ }^{3,4}$ \\ 1Department of Chemical Engineering, Polytechnic University of Catalonia - BarcelonaTech, C/ Eduard Maristany 10- \\ 14 (Campus Diagonal-Besòs), 08930, Barcelona, Spain \\ 2Barcelona Research Center in Multiscale Science and Engineering, C/ Eduard Maristany, 10-14 (Campus Diagonal- \\ Besòs), 08930, Barcelona, Spain \\ ${ }^{3}$ Department of Chemical Engineering, Polytechnic University of Catalonia - BarcelonaTech, av. Diagonal 647, 08028 \\ Barcelona, Spain \\ ${ }^{4}$ ICREA, Passeig Lluís Companys 23, Barcelona, Spain
}

\begin{abstract}
A better understanding of ion-transport mechanisms requires separate information on the equilibrium (partitioning) and kinetic (diffusivity) properties of the membranes with respect to ions. This work presents a novel non-stationary-diffusion method to determine salt diffusion and partitioning coefficients under (quasi)-linear conditions of relatively small concentration differences.
\end{abstract}

An ion-exchange membrane supported by a relatively thick coarse-porous support (glass frit) is placed in a two-compartment stirred cell. The salt concentration in one compartment is kept stationary during the measurement whereas in the other compartment, the initial solution is rapidly replaced by a solution of different concentration. As a result, there is a time-dependent electrical response due to a progressive redistribution of applied concentration difference between the membrane and the porous support and the different ion perm-selectivities of those media. A mathematical model is developed to interpret the data. The rate of signal relaxation is primarily controlled by the diffusion permeability of the membrane but is also affected by the salt partitioning. In addition, osmotic trans-membrane volume transfer has a significant impact on the relaxation process, so it needs to be taken into account. The osmotic permeability has been determined in separate measurements.

Systematic studies have been carried out at various $\mathrm{NaCl}$ concentrations with Nafion 120 and type 10-Fujifilm ion-exchange membranes. The results obtained for Nafion 120 are in agreement with the literature data. This approach allows for a relatively simple determination of salt permeability and partitioning coefficient in addition to the ion perm-selectivity, which is the only parameter available from the conventional measurements of stationary membrane potential.

Keywords: ion-exchange membrane; transient membrane potential; concentration step; ion partitioning; salt diffusion permeability 


\section{Introduction}

Ion-exchange membranes are central elements of electro-membrane processes [1-7]. For their optimization, it is important to have detailed information on the transport and equilibrium properties of ion-exchange membranes, in particular, separate information on the equilibrium (partitioning) and kinetic (diffusivity) properties of the membranes with respect to ions. The partition coefficient of an ion refers to its distribution between the membrane and the solution whereas the diffusivity coefficient is related to the ion mobility in the membrane [8].

Stationary techniques of membrane characterization such as membrane potential or DC electrical resistance provide only information on ionic permeabilities, which are products of partitioning and diffusion coefficients [9]. As the steady state permeability involves both properties, by knowing one of them the other can be calculated [10].

Equilibrium salt sorption can be measured via salt desorption using standard methods or advanced techniques such as attenuated total reflection Fourier transform infrared spectroscopy (ATR-FTIR) [11-14], Rutherford backscattering spectrometry (RBS) $[15,16]$ or quartz crystal microbalance (QCM) sensors [17]. On the other hand, diffusivity may be measured using tracer-based techniques such as pulsed-field gradient nuclear magnetic resonance (PFG-NMR) [18,19]. Moreover, electrochemical impedance spectroscopy (EIS) has been used to determine both partitioning and diffusivity coefficients in the case of electroactive solutes [20].

Non-stationary diffusion in principle allows for separate determination of those properties for a diffusing species from interpretation of a single time-resolved measurement [21]. However, the typical use of pure solvent in the receiving compartment (needed to reliably detect initially small concentration changes) [22] implies large trans-membrane salt-concentration differences and strongly non-linear diffusion that would complicate the interpretation in the case of ion-exchange membranes whose diffusion permeability is a strong function of salt concentration. Nonstationary diffusion of radiotracer ions $[8,23]$ can provide information on the ion diffusivity and partitioning from which the salt-related properties can be calculated. However, the use of radiotracers is possible only in certified laboratories. Besides, suitable radiotracers are available only for some ions.

A different approach based on time-resolved measurements consists in measuring the electrical response to a sudden change in the electrolyte concentration at one of the membrane sides [24-26]. Sørensen and Compañ developed a method to determine ion transport numbers within a surface layer of a membrane by measuring initial-time membrane potential. Once the membrane was equilibrated with an equilibrium solution, one of the membrane faces was exposed to a non-equilibrium solution and the membrane potential was measured immediately. Initially, the concentration gradient 
remained localized within a surface layer of the membrane. Therefore, the electrical response was controlled by the ion transport numbers within a narrow surface zone and could be estimated for each face of the membrane. The initial time method was used to evaluate the asymmetry between the two faces of a membrane and conclude if it was homogeneous or not.

Later, refs. $[27,28]$ pointed out a problem related to the solution replacement technique (as implemented in [24-26]), which is the presence of an unstirred layer at the membrane surface that does not allow for a change in the electrolyte concentration directly at the membrane surface but only at the external surface of a boundary layer. For nanofiltration membranes studied in $[27,28]$ the characteristic relaxation time of the initial signal could be as short as a couple of milliseconds, so in the presence of an unstirred layer it was impossible to measure the initial membrane-potential values. Therefore, refs.[27,28] introduced an alternative approach to the rapid concentration change at the membrane surface (touching the membrane surface with a pendant drop).

However, in the case of ion-exchange membranes, the relaxation of initial signal takes tens of seconds since these membranes are relatively thick compared with the active layers of nanofiltration membranes. Therefore, the solution replacement can be implemented simply via evacuating an equilibrium solution and replacing it with a nonequilibrium one.

In this study, the electrical response to this is measured for a membrane supported by a relatively coarse-porous material. The different ion perm-selectivities of the membrane (thin and relatively dense) and porous support (thicker and much more porous than the membrane) make the response time-dependent due to the progressive redistribution of applied concentration difference between those two media. The porous support was characterized in separate measurements in terms of porosity and effective salt diffusivity needed for the interpretation of the results. Experimental data is fitted to a mathematical model that describes transient transport phenomena including osmosis, which has noticeable impact on the measurements (see Supporting information for detailed information.

First, the new procedure is applied to a membrane (Nafion 120) that has been previously extensively characterized by using well-established conventional techniques. The results will be compared with the literature to validate the new method. Further on, the method will be implemented for a range of $\mathrm{NaCl}$ concentrations to obtain information on concentration dependences of properties of a novel ion-exchange membrane (Type 10, Fujifilm NL). 


\section{Theory}

The present work considers a system consisting of a membrane and a porous support placed in a two-compartment cell. Initially, both compartments are filled with a base solution of concentration $c_{0}$; the membrane and the porous support are equilibrated with this solution. Suddenly, the solution facing the membrane is replaced by a solution of a different concentration, $c_{\text {step }}$.

Fig.1 shows an example of non-stationary concentration profile occurring some time after a concentration step in a system formed by a membrane and a porous support as described. $\mathrm{L}_{\mathrm{m}}, \mathrm{L}_{s}$ are the thicknesses of membrane and porous support respectively. $c_{i}$ is the salt concentration at the membrane/porous support interface.

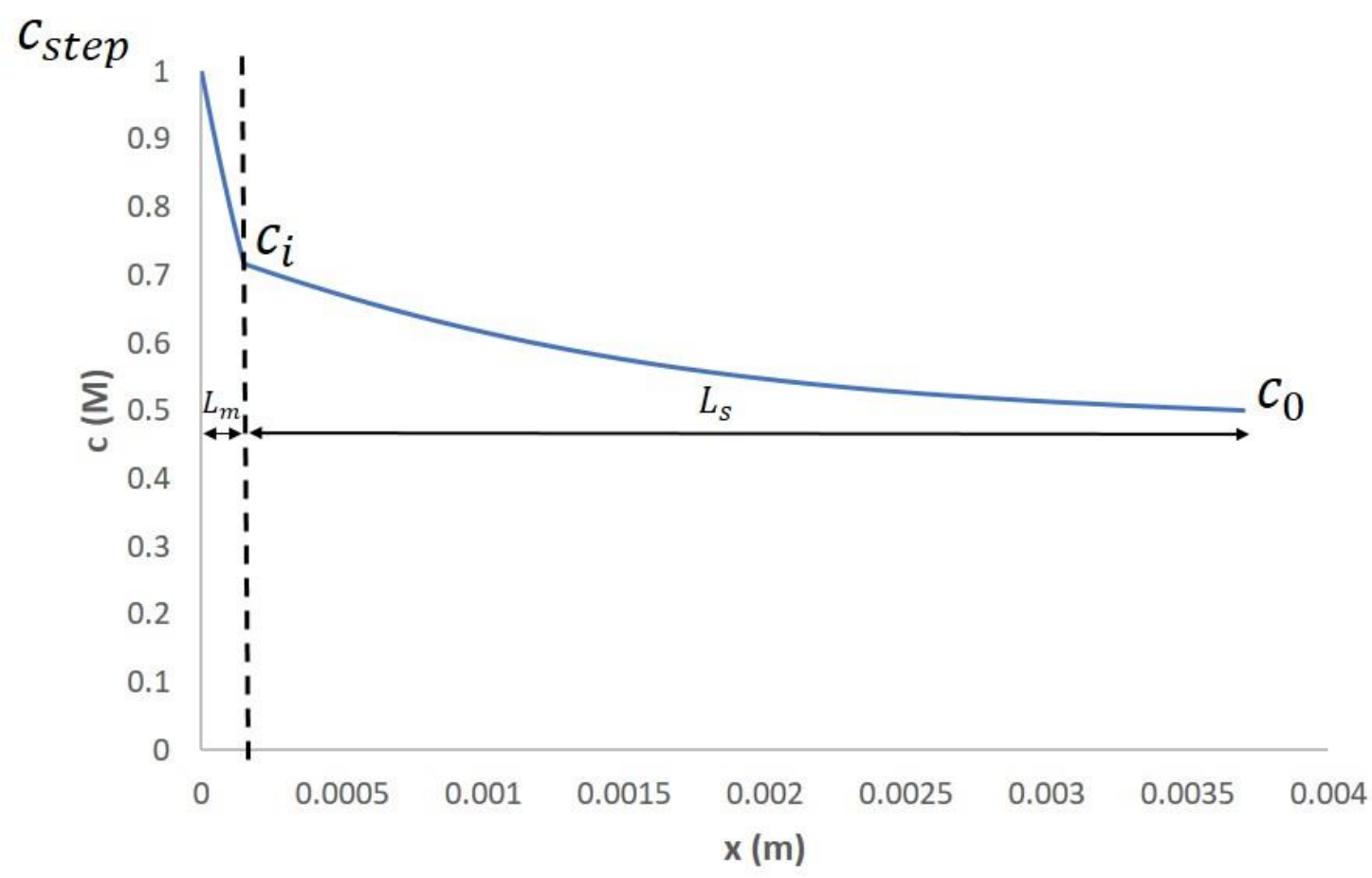

Fig.1 Non-stationary salt-concentration profile

The diffusion process occurs through a large chemical resistance (thin and relatively dense ion-exchange membrane) into a large chemical capacity (the porous support), which is much more porous and thicker than the membrane. In this way, there is a progressive redistribution of applied concentration difference between the membrane and the porous support. Due to the different ion perm-selectivities of those media this gives rise to a time-dependent electrical response. 
Another important phenomenon occurring in the system is osmosis. Some estimates concerning the influence of osmosis are presented in Supporting information. There is an osmotic flow, $J_{v}$, transporting water in the opposite direction to the salt transport:

$J_{v}=-K *\left(c_{\text {step }}-c_{i}\right)$

where $K$ is the osmotic permeability defined as:

$K=v R T \chi\left(1-T_{m}\right)$

where $v$ is the stoichiometric coefficient, $R$ is the gas constant, $T$ is the absolute temperature, $T_{m}$ is the transmission coefficient and $\chi$ is the mechanical permeance.

Taking into account both transport phenomena, the salt mass balance in the membrane is described by:

$\alpha_{m} \frac{\partial c_{m}}{\partial t}=\frac{\partial}{\partial \mathrm{x}}\left[-D_{m} \cdot\left(1+\frac{d \ln (\gamma)}{\operatorname{dln}\left(c_{m}\right)}\right) \cdot \frac{\partial c_{m}}{\partial x}+J_{v} T_{m} c_{m}\right]$

where $\alpha_{m}$ is the chemical capacity in the membrane, $c_{m}$ is the virtual salt concentration [29] in the membrane, $D_{m}$ is the salt diffusivity in the membrane, $\gamma$ is the salt activity coefficient in the virtual solution, $x$ is the longitudinal coordinate $\left(0<x<L_{m}\right)$ and $T_{m}$ is the salt transmission coefficient.

The chemical capacity is a quantitative measure of how much salt has to be added to a unit of volume of a medium to change the salt chemical potential by a unit [30]. This property is related to the partition coefficient of either of the ions, $\Gamma_{ \pm}$as

$\alpha_{m}=\Gamma_{ \pm}\left(1+\frac{d \ln \Gamma_{ \pm}}{d \ln c}\right)$

It can be shown that due to the electroneutrality of membrane phase using either of ions gives the same result.

Eq. (3) has to be solved numerically with the following initial and boundary conditions:

$\left.c_{m}\right|_{x=0}=c_{\text {step }} * \frac{1}{1+e^{-2 \pi \cdot\left(\frac{2}{t_{\text {step }}} t-1\right)}}$

$\left.c_{m}\right|_{x=L_{m}}=c_{i}$

The first of these conditions approximates numerically the concentration step in the non-equilibrium compartment, where $t_{\text {step }}$ is the numerical step duration, assumed to be $0.001 \mathrm{~s}$ in the present work.

For the salt transport in the porous support, a similar equation is used: 
$\alpha_{s} \frac{\partial c_{s}}{\partial t}=\frac{\partial}{\partial \mathrm{x}}\left[-D_{s} \cdot\left(1+\frac{d \ln (\gamma)}{\operatorname{dln}\left(c_{s}\right)}\right) \cdot \frac{\partial c_{s}}{\partial x}+J_{v} c_{s}\right]$

In this case $\alpha_{s}$ is the chemical capacity in the porous support (which is equal to the porosity), $c_{S}$ is the salt concentration in the porous support, $D_{s}$ is the salt diffusivity in the porous support, $x$ is the longitudinal coordinate $\left(L_{m}<x<\left(L_{m}+L_{s}\right)\right)$ and the corresponding boundary conditions are:

$\left.c_{S}\right|_{x=L_{m}}=c_{i}$

$\left.c_{s}\right|_{x=\left(L_{m}+L_{s}\right)}=c_{0}$

Eqs. (3) and (6) are a system of partial differential equations (PDE) that can be solved numerically and the evolution of electric potential difference, $\varphi$, is given by [31] :

$\varphi=\frac{R T}{F}\left[\left(2 t_{+}^{m}-1\right) \ln \left(\frac{a_{\text {step }}}{a_{i}}\right)+\left(2 t_{+}^{b}-1\right) \ln \left(\frac{a_{i}}{a_{0}}\right)\right]$

where $R$ is the ideal gas constant, $T$ is the absolute temperature, $F$ is the Faraday constant, $a$ is the activity, $t_{+}^{b}$ is the transport number of cations in the porous support and $t_{+}^{m}$ is the transport number of cations in the membrane. The transport number of cations in the porous support is assumed to be equal to the transport number in the bulk electrolyte solution:

$t_{+}^{b}=\frac{D_{+}}{D_{+}+D_{-}}$

Initially the whole concentration gradient is located on the membrane. Therefore, the transport number of positive ions in the membrane, $t_{+}^{m}$, can be estimated from:

$t_{+}^{m}=\frac{1}{2}\left[\frac{\frac{F \varphi_{0}}{R T}}{\ln \left(\frac{c_{\text {step }} \gamma_{\text {step }}}{c_{0} \gamma_{0}}\right)}+1\right]$

where $\varphi_{0}$ is the initial value of electric-potential difference occurring just after the solution replacement.

The described model has been validated using several approaches (see Supporting information). 


\section{Experimental materials and methods}

\section{Materials}

Experimental data have been obtained with two different ion-exchange membranes: Nafion 120 (DuPont) and Type 10 kindly provided by Fujifilm Manufacturing Europe BV (The Netherlands). Membranes were supported by porous glass frit discs of $25 \mathrm{~mm}$ in diameter having a non-porous peripheral edge (the porous part is $19 \mathrm{~mm}$ in diameter, see Fig. 2b), $3.65 \mathrm{~mm}$ of thickness and average pore size of $10-16 \mu \mathrm{m}$ supplied by Duran Group (Germany). Electric potential difference was measured with $\mathrm{Ag} / \mathrm{AgCl}$ reference electrodes with salt bridges filled with $\mathrm{KCl} 3 \mathrm{M}$ (Metrohm, Switzerland). The chemical reagents were of analysis grade.

\section{Measurements of transient-membrane potential after concentration step}

Nafion 120 membranes were conditioned in boiling water for $30 \mathrm{~min}$. Membranes were equilibrated overnight with the solution of base concentration, $\mathrm{C}_{0}$. Equilibrated sample is placed along with the porous support in a two-compartment cell (Fig. 2a). All the measurements were performed at room temperature. At the start, the two compartments are filled with the base solution and a measuring electrode is located in each compartment. The salt concentration on the porous support side (equilibrium compartment) is kept constant during the measurement. Once the measured electric potential between the two compartments is constant, the solution in the compartment facing the membrane is replaced by the non-equilibrium solution of concentration $C_{\text {step }}$. The equilibrium solution is sucked out by a syringe, a process that takes typically around $10 \mathrm{~s}$, whereas the new solution is just poured from a beaker. Measurements were performed for several base concentrations and the ratio $C_{\text {step }}$ to $C_{0}$ was 2 in all cases. The solution in the non-equilibrium compartment was stirred using a magnetic stirrer to keep the concentration at the membrane surface constant.

(a)

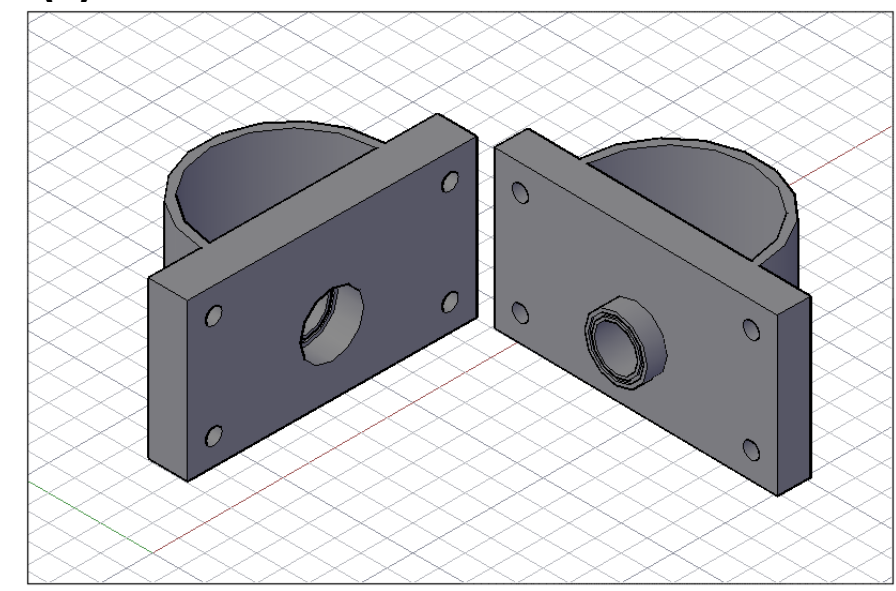

non-porous peripheral ring

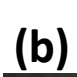

(b)

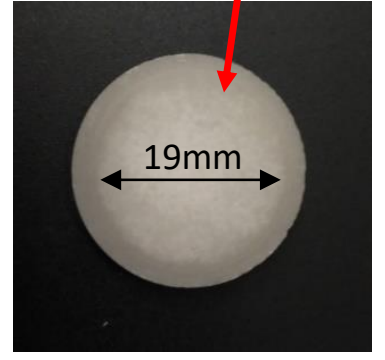


Fig. 2 a) Drawing of the cell and b) glass frit used as a porous support

\section{Measurement of osmotic permeability}

Osmotic permeability was determined for the Type 10 Fujifilm membranes only. A membrane disk of $25 \mathrm{~mm}$ in diameter is placed in a two-compartment stirred cell. One compartment (contains the more dilute solution) is open and the other one is closed and equipped with a graduated pipette to measure changes in the volume as a function of time due to the osmotic flow. The membrane was equilibrated overnight with a solution having concentration between dilute and higher-concentration solutions. Solutions were pre-heated moderately in order to remove dissolved air to avoid formation of bubbles during the measurement.

The slope of linear dependence of half-cell volume on time gives the osmotic flux, $J_{v}$. The osmotic permeability, $K$, was calculated as the ratio of osmotic flux and corrected concentration difference taking into account deviations from solution ideality by means of osmotic coefficient, $\phi$ :

$K=\frac{J_{v}}{C_{2} \cdot \phi_{2}-C_{1} \cdot \phi_{1}}$

where $c$ is the salt concentration and the subscripts 1 and 2 denote the lower and higher concentration respectively.

The osmotic permeability was determined for the same concentration pairs $C_{0}-C_{\text {step }}$ as used in the transient membrane potential measurements.

\section{Glass frit characterization}

For the interpretation of measurements of transient membrane potential, we need to know the porosity of and effective salt diffusion coefficient in the glass frit used as the porous support. They were determined in the following way.

First, the porosity was determined gravimetrically. A glass frit was immersed in distilled water, water from the surface was removed and the mass of the wet disc was recorded. Then, the disc was dried in an oven until the mass of the disc was stable. This procedure was repeated four times and the result is the average of the measurements. The porosity was calculated as:

porosity $=\frac{\left(m_{w}-m_{d}\right)}{\frac{\pi}{4} \cdot d_{f}^{2} \cdot \delta \cdot \rho_{w}}$

where $m_{w}$ and $m_{d}$ are the wet and dry masses respectively, $d_{f}$ is the diameter of the porous part, $\delta$ is the thickness and $\rho_{w}$ is water density. 
The effective salt diffusion coefficient in the glass frit was estimated indirectly via AC electrical resistance. The measured resistance was compared to the electrical resistance of free solution of the same geometry calculated as

$R_{\text {electrolyte }}=\frac{L}{\sigma \cdot A}$

where $A$ is the cross-sectional area of the porous part, $L$ is the thickness and $\sigma$ is the conductivity. It is assumed that diffusivity reduction factor is the same as conductivity reduction.

A glass frit disc was sandwiched between two pieces of a stainless steel net (Dexmet corporation, USA) used as electrodes and the AC electrical resistance was measured with a sourcemeter (2400 Series SourceMeter, Keithley Instruments).

\section{Modelling procedure}

The procedure to fit the experimental data to the model described above is as follows.

1. Obtain the values of diffusion coefficient for each ion $\left(D_{+}, D_{-}\right)$and the activity coefficient $\left(\gamma_{0}, \gamma_{\text {step }}\right)$

2. Determine the transport numbers in the membrane $\left(t_{+}^{m}\right)$ and in the bulk solution $\left(t_{+}^{b}\right)$ using Eqs. (9), (10) respectively

3. Solve numerically the PDE system formed by Eqs. (3), (6). In this work, MATLAB and pdepe function to solve initial-boundary value problems for systems of parabolic and elliptic PDEs has been used. The concentration dependence of salt activity coefficient was taken from the literature [32]. The necessary values of $\alpha_{s}, D_{s}, L_{s}$ were determined from the porous-support characterization, $L_{m}$ was measured with a digital caliper and $K$ was obtained from the osmotic permeability measurements. The values of $\alpha_{m}, D_{m}, T_{m}$ are initially guessed to start the resolution

4. A time-dependence of $c_{i}$ is obtained from the previous step and the activity coefficient $\gamma_{i}$ is determined for each time. This concentration time-dependence is converted in membrane potential $\varphi_{\text {model }}$ using Eq. (8)

5. The function $\varphi_{\text {model }}$ is compared with the time-dependence potential obtained experimentally, $\varphi_{\text {exp }}$. The model parameters $\alpha_{m}, D_{m}, T_{m}$ are iteratively changed until the percentage difference between both values is below $1 \%$

6. Finally, partitioning coefficient $\Gamma_{ \pm}$can be calculated from chemical capacity $\alpha_{m}$ by means of Eq. (4) 


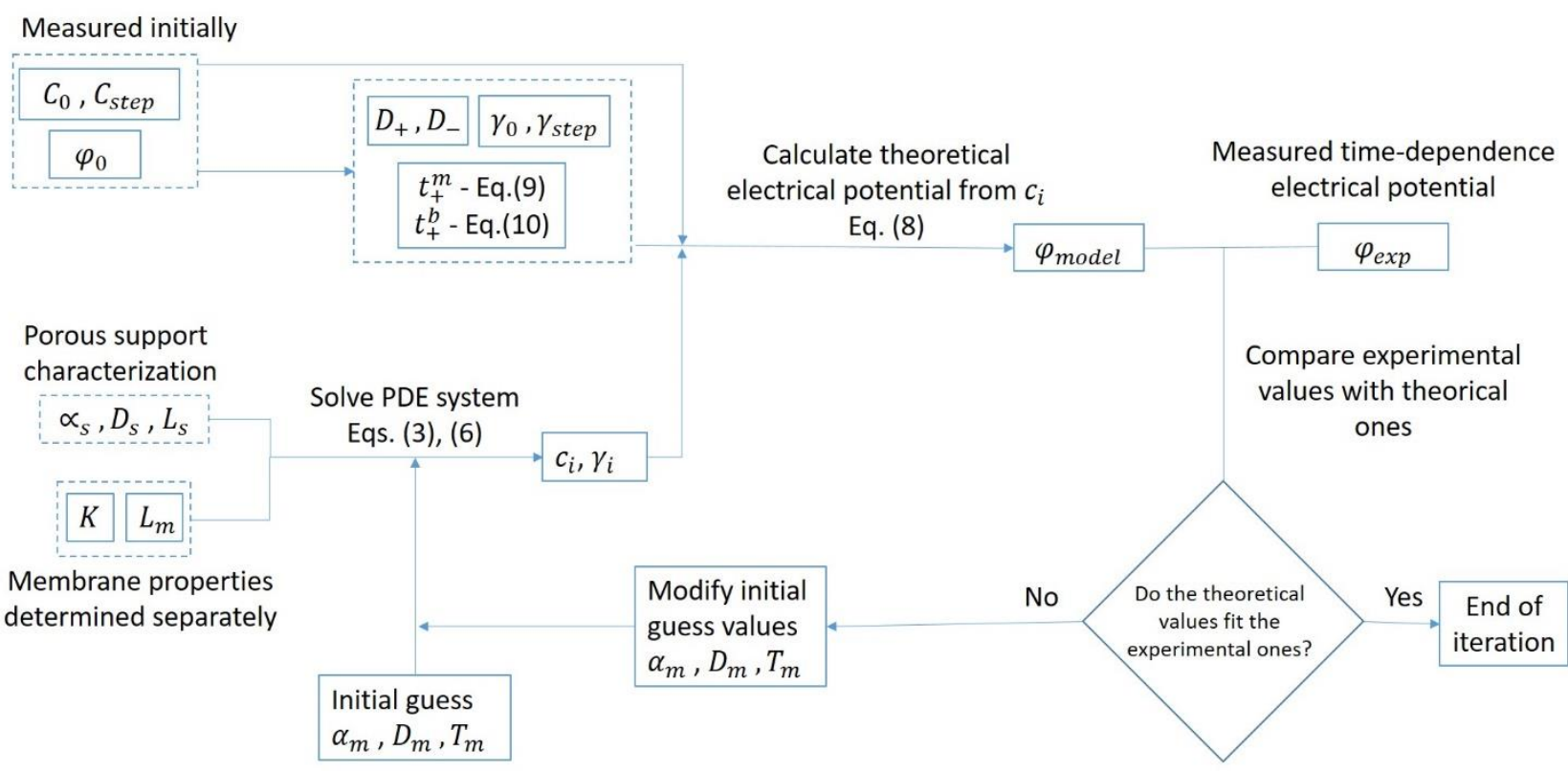

Fig. 3 Modelling procedure diagram to obtain model parameters $\boldsymbol{\alpha}_{m}, \boldsymbol{D}_{m}, \boldsymbol{T}_{m}$ from the experimental data

\section{Results and discussion}

\section{Glass frit properties}

Table 1 shows the properties of the glass frit. In the case of diffusion coefficient estimation in the glass frit, it was assumed that the modification in its electrical resistance compared to the free solution layer of the same geometry is the same as the increase in its diffusion resistivity. This assumption is quite realistic taking into account the relatively large pore size of the glass frit. The ratio between glass frit resistance to free solution resistance was $10.68 \pm 0.26$. Therefore, taking into account the salt diffusivity of $\mathrm{NaCl}$ solution $\left(1.62 \cdot 10^{-9} \mathrm{~m}^{2} / \mathrm{s}\right)$ the effective diffusion coefficient in the glass frit was set at $1.5 \cdot 10^{-10} \mathrm{~m}^{2} / \mathrm{s}$.

Table 1

Properties of porous support

\begin{tabular}{cccc}
\hline$\alpha_{s}$ & $D_{s}\left(\mathrm{~m}^{2} / \mathrm{s}\right)$ & Pore size $(\mu \mathrm{m})$ & $L_{s}(\mathrm{~mm})$ \\
\hline $0.34 \pm 0.00$ & $1.5 \cdot 10^{-10}$ & $10-16$ & 3.7 \\
\hline
\end{tabular}




\section{Osmotic permeability determination}

Fig.4 shows a typical time evolution of volume transferred by osmosis.

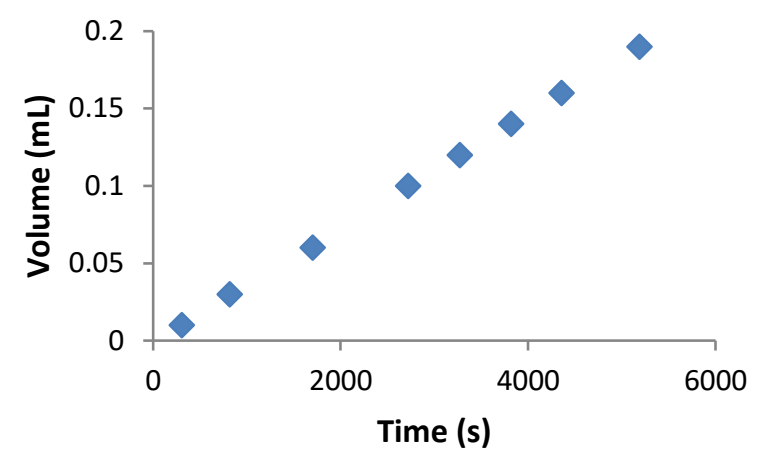

Fig.4 Time dependence of closed half-cell volume in measurements of osmosis using CEM type 10 membrane and $1 \mathrm{M}-2 \mathrm{M} \mathrm{NaCl}$

The slope of volume vs. time dependence gives the osmotic flux, which has been determined for both anion and cation exchange membranes for a concentration range always using the same concentration pairs as in the measurements of transient membrane potential.

Fig. 5 shows the osmotic flux as a function of lower concentration. Expectedly, the higher is the concentration, the larger is the osmotic flux. It can also be seen that the osmosis is noticeably stronger in CEM than in AEM.

(a)

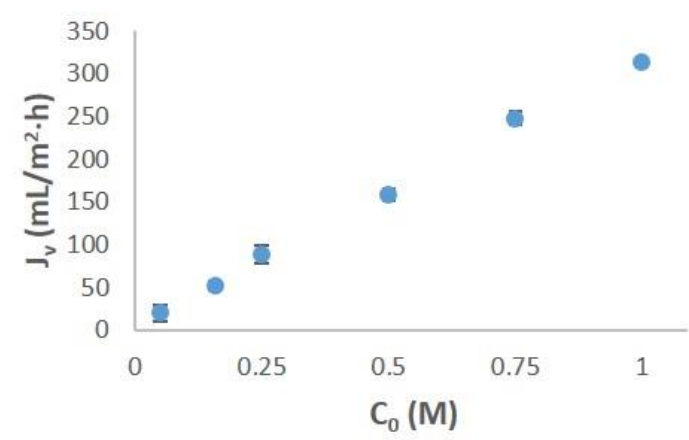

(b)

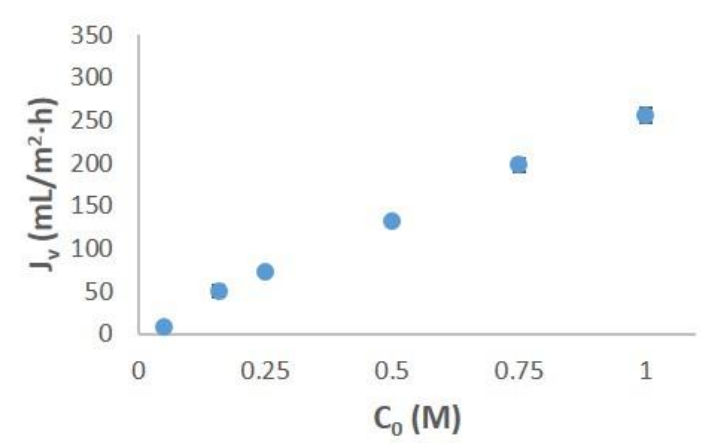

Fig. 5 Osmotic flux vs. lower concentration determined for Type 10 Fujifilm: a) CEM and b) AEM 
Table 2 presents the osmotic permeability calculated as the ratio between the measured osmotic flow and the concentration difference (corrected for the solution non-ideality). These values have been used in the model validation (see previous section) and will be used for the fitting of time dependences of transient membrane potential below.

\section{Table 2}

Osmotic permeability of cation and anion exchange membrane type 10. $C_{0}$ is the lower concentration and the higher concentration is two times larger.

\begin{tabular}{ccc}
\hline$C_{0}$ & \multicolumn{2}{c}{$K \cdot 10^{-10}\left(\mathrm{~m}^{4} / \mathrm{mol} \cdot \mathrm{s}\right)$} \\
\cline { 2 - 3 }$(\mathrm{M})$ & $\mathrm{CEM}$ & $\mathrm{AEM}$ \\
\hline 0.05 & $1.25 \pm 0.59$ & $0.47 \pm 0.20$ \\
0.158 & $1.02 \pm 0.11$ & $0.98 \pm 0.14$ \\
0.25 & $1.46 \pm 0.16$ & $1.21 \pm 0.06$ \\
0.5 & $0.93 \pm 0.04$ & $0.77 \pm 0.02$ \\
0.75 & $0.66 \pm 0.02$ & $0.53 \pm 0.02$ \\
1 & $0.84 \pm 0.0005$ & $0.69 \pm 0.02$ \\
\hline
\end{tabular}

Estimates of effective salt diffusion and partitioning coefficients

Fig.6 shows a typical example of measurement of transient-membrane potential after concentration step with Nafion 120 membrane. All the presented results are averages of at least three measurements. 


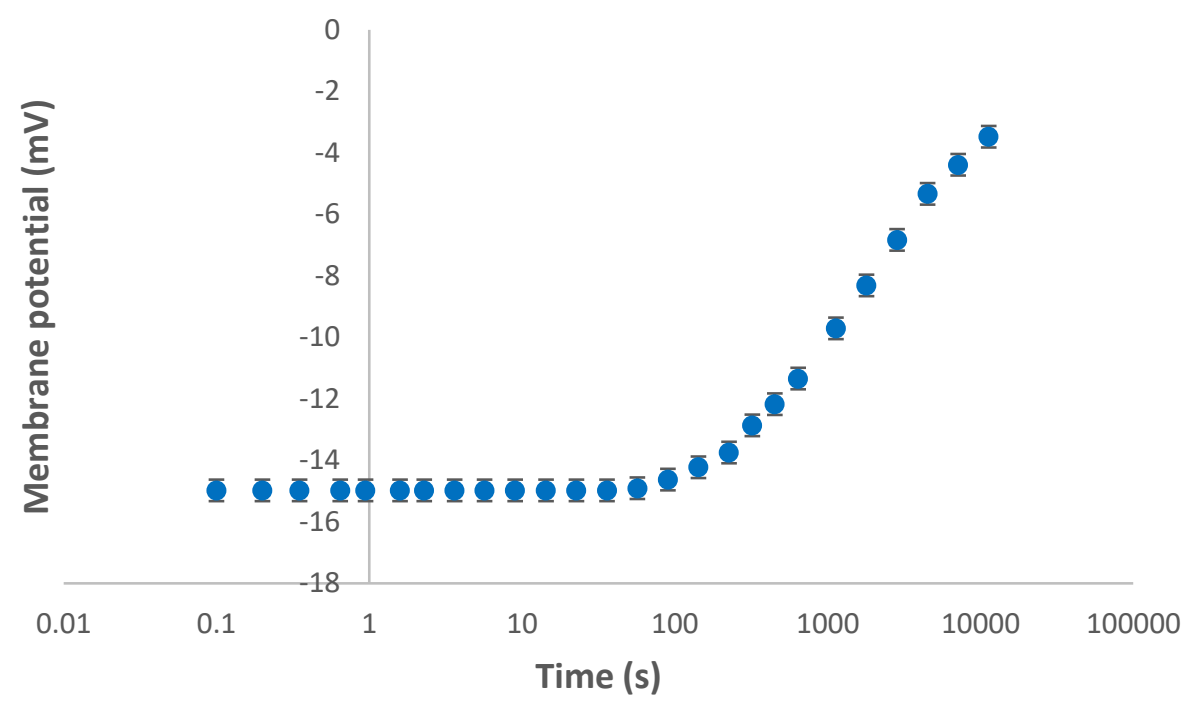

Fig. 6 Experimental time evolution of membrane potential for Nafion $120, \boldsymbol{C}_{\mathbf{0}}=\mathbf{0} . \mathbf{5} \boldsymbol{M}$ $\mathrm{NaCl}$

As can be seen from Fig.6, when the solute concentration is suddenly changed at the membrane surface, an initial potential difference remains practically constant for a certain period of time. This is because in macroscopically homogeneous membranes transmembrane electric-potential difference is independent of shape of saltconcentration profile. Accordingly, the signal evolution begins only when the salt concentration at the interface starts to change. The diffusion occurs through a large chemical resistance, which is the thin and relatively dense ion-exchange membrane, into a large chemical capacity, namely the porous support, which is much more porous and thicker than the membrane. The salt chemical potential at the membrane/support interface remains practically constant until the chemical capacity of the adjacent part of the support is noticeably charged [28]. This explains why the initial period of constant signal is relatively long.

After that, the response becomes time-dependent because of progressive redistribution of applied concentration difference between the membrane and the porous support and the different ion perm-selectivities of those media. The pattern of signal relaxation is primarily controlled by the diffusion permeability of the membrane but is also affected by the salt partitioning coefficient. In addition, osmotic trans-membrane volume transfer has a considerable impact on the relaxation process (see Supporting information for detailed information).

From the initially-constant signal one can determine the ionic perm-selectivity of the membrane according to the definition [33]: 
permselectivity $=\frac{t_{\text {counter-ion }}^{m}-t_{\text {counter-ion }}^{b}}{t_{\text {co-ion }}^{b}}$

where $t$ is the transport number calculated from the initial membrane potential with Eq. (10). The superscripts $m, b$ refer to the membrane and bulk-solution phases. Measurements are performed for Nafion 120 membranes at different values of base concentration $C_{0}$ and effective salt diffusion and partition coefficients are determined by fitting the experimental data to the mathematical model as described above (see Fig.3). The osmotic permeability was estimated from the osmotic flow data published by Narebska et al [34]. The obtained results will be compared with the literature to validate the suggested procedure.

Fig. 7 shows the experimental data collected at several base solution concentration $C_{0}$. The ratio $C_{\text {step }} / C_{0}$ was kept at 2 in all the measurements. Each individual measurement was fitted separately and the averages of the obtained fitted parameters for each base concentration are presented as the results. Fig. 7 also presents the theoretical curves obtained in each case. It illustrates mostly excellent quality of the theoretical fits. It can also be seen that the initial membrane potential decreases (in absolute value) with concentration, which indicates that the electrochemical perm-selectivity decreases with concentration. This has been typically observed for ion-exchange membranes [27].

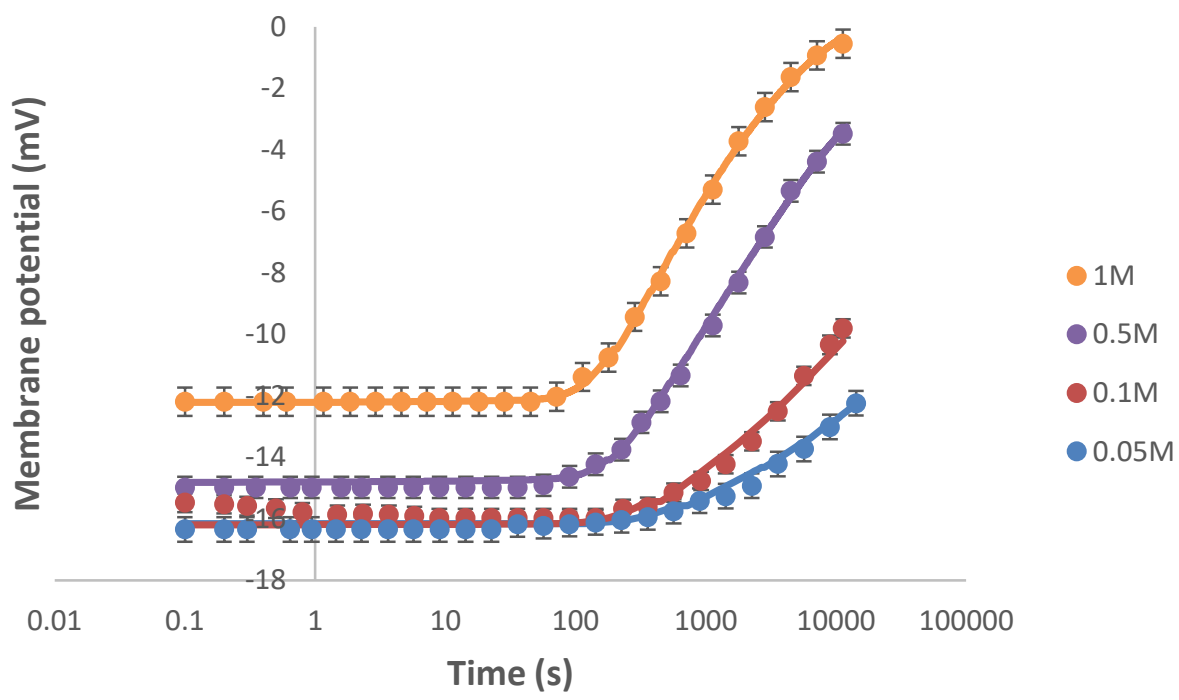

Fig. 7 Time evolution of trans-membrane potential for various base concentrations, $C_{0}$, for Nafion 120. $\boldsymbol{C}_{\text {step }}$ is always two times higher than $\boldsymbol{C}_{\mathbf{0}}$. Dots represent experimental data and solid lines are the theoretical fits

The average values of partition and effective diffusion coefficients obtained from the fitting are shown in 
Fig. 8 and compared with the data published in the literature [34,35]. In all cases the results are quite similar, so the presented method is validated.
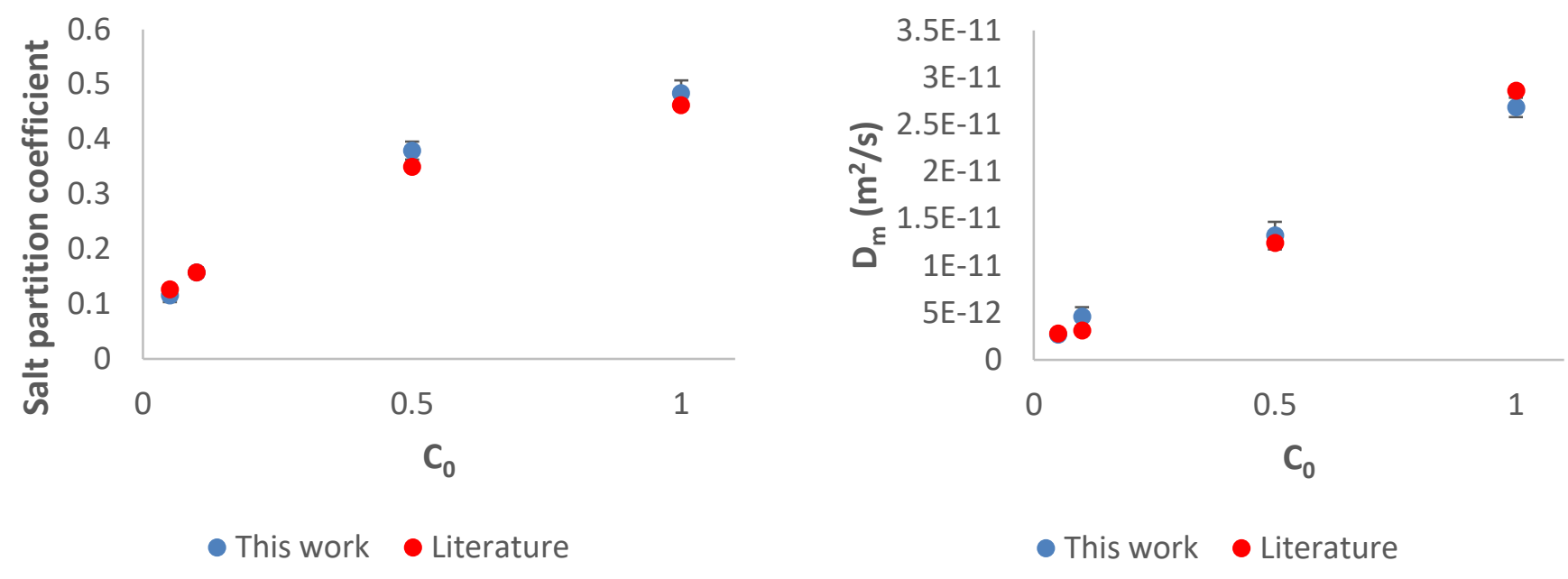

Fig. 8 Partition and diffusion coefficients obtained in this work for Nafion 120 membrane compared with the literature $[34,35]$

Now, the same procedure is applied to two different novel membranes, one cation- and another anion-exchange. Sample measurements for both type 10 Fujifilm membranes are presented in

Fig.9. CEM type 10 shows a behaviour quite similar to Nafion 120 whereas AEM type 10 exhibits signal of opposite sign, which is due to the opposite (positive) sign of fixed charge. 


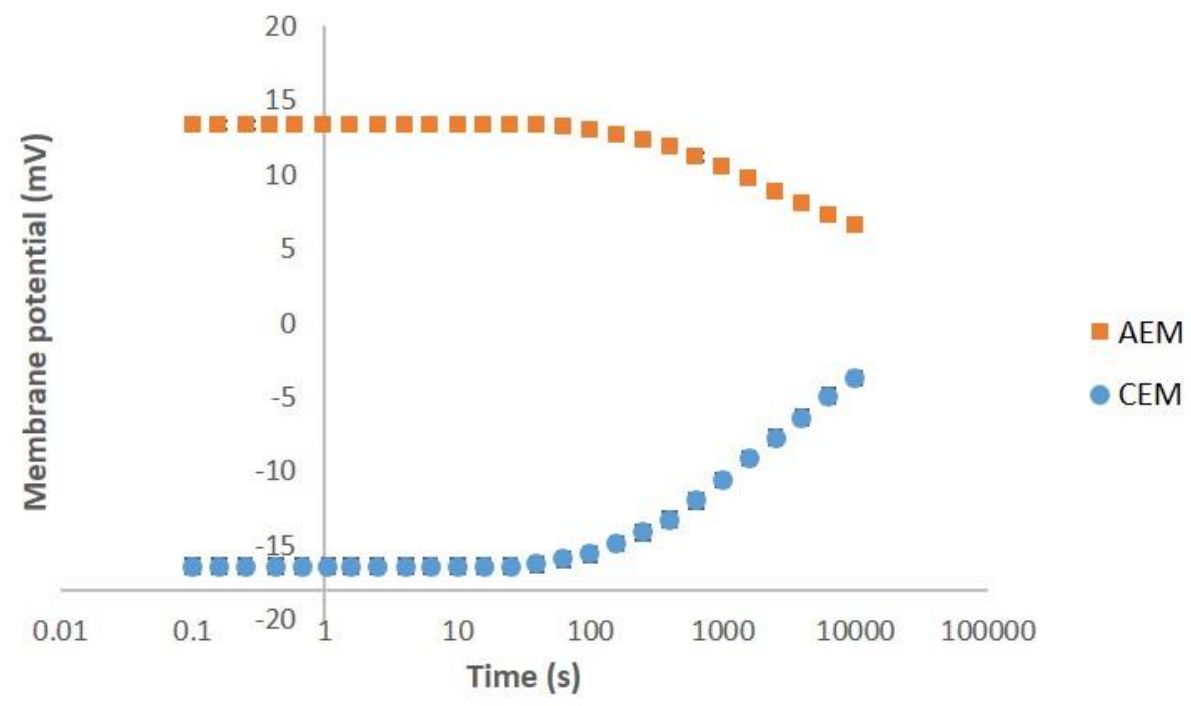

Fig.9 Experimental time evolution of membrane potential for type 10 Fujifilm cation and anion exchange membranes for $\boldsymbol{C}_{\mathbf{0}}=\mathbf{0 . 5} \mathbf{M ~ N a C l}$

The effect of variation of base concentration, $C_{0}$, has been studied in the concentration range between $0.05 \mathrm{M}$ and $1 \mathrm{M} \mathrm{NaCl}$ keeping again $C_{\text {step }}$ always two times higher than the base solution concentration $C_{0}$ and the results are presented in Fig.10. Along with the experimental data, Fig.10 also shows the theoretical fits obtained for each curve, whose quality is quite good in all the cases. It also can be seen that the initial membrane potential decreases (in absolute value) with concentration but this dependence is relatively weak in the case of CEM, whereas for AEM it is stronger. This decrease has also been observed in the measurements with Nafion 120, which is due to the decrease in electrochemical perm-selectivity with concentration observed in ion-exchange membranes as explained above. This is reflected in the counter-ion transport numbers presented in Table 3. 
(a)

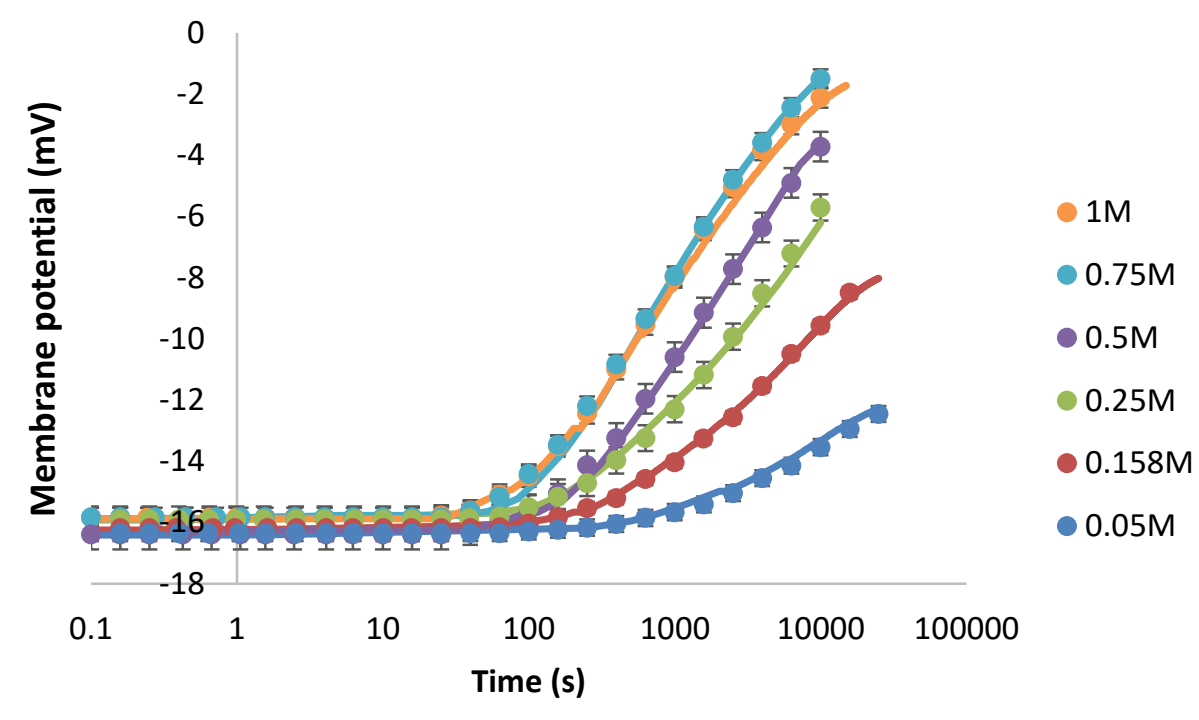

(b)

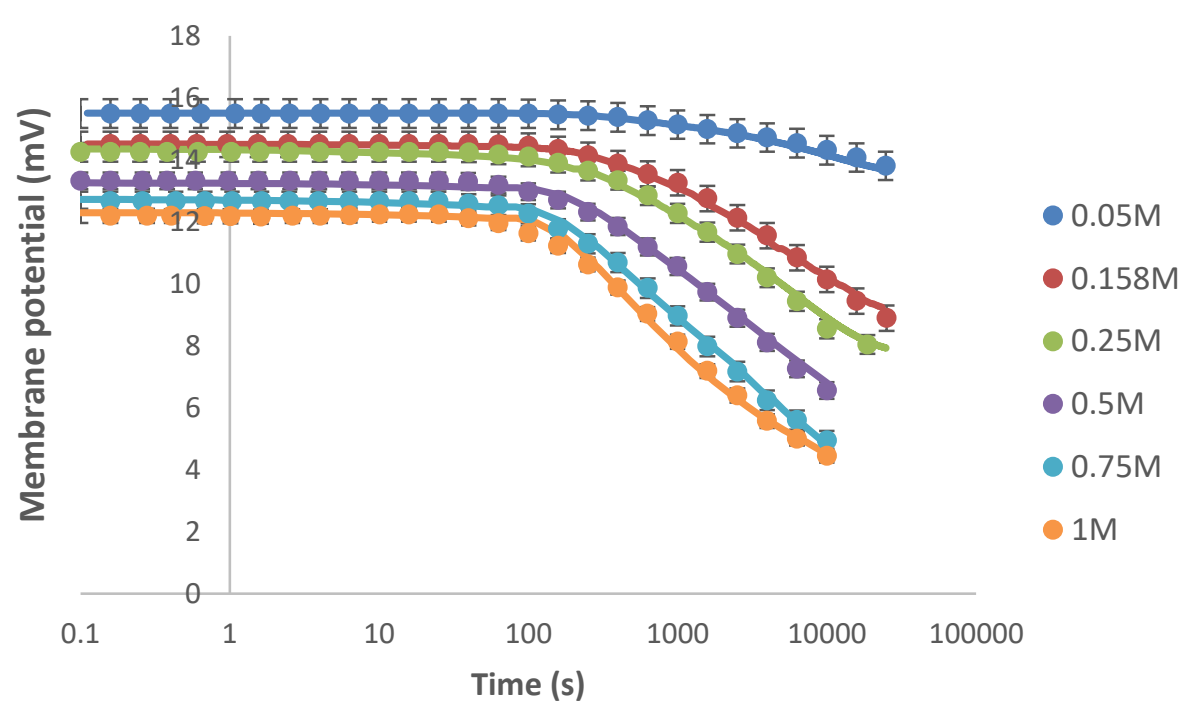

Fig.10 Time evolution of trans-membrane potential for various base concentrations, $\mathrm{C}_{0}$ , for Type 10 Fujifilm membranes: a) CEM and b) AEM. $\boldsymbol{C}_{\text {step }}$ is always two times higher than $\boldsymbol{C}_{\mathbf{0}}$. Symbols represent experimental data and solid lines are the theoretical fits

\section{Table 3}

Counter-ion transport number in the membrane for CEM/AEM type 10

\begin{tabular}{|c|c|c|}
\hline \multirow{2}{*}{$\mathrm{C}_{0}(\mathrm{M})$} & \multicolumn{2}{|c|}{$t_{\text {counter-ion }}^{m}$} \\
\hline & CEM & AEM \\
\hline 0.05 & $1.007 \pm 0.007$ & $0.979 \pm 0.003$ \\
\hline 0.158 & $1.002 \pm 0.003$ & $0.948 \pm 0.008$ \\
\hline 0.25 & $0.978 \pm 0.011$ & $0.939 \pm 0.009$ \\
\hline 0.5 & $0.985 \pm 0.005$ & $0.896 \pm 0.004$ \\
\hline 0.75 & $0.959 \pm 0.004$ & $0.867 \pm 0.006$ \\
\hline
\end{tabular}


Fig.10 also shows that the characteristic relaxation time decreases when the base concentration increases. A shorter relaxation time implies higher diffusion permeability, so this should increase with the salt concentration.

The values of partition, effective salt diffusion and transmission coefficients and permselectivity obtained from the fitting are shown in Fig.11 for CEM and in Fig.12 for AEM. In both cases there are increasing trends in the partition and effective salt diffusion coefficients with the base concentration, which is in agreement with the Donnan exclusion of co-ions from ion-exchange materials. The same can be said about the decreasing trend of perm-selectivity. It is also seen that the perm-selectivity for CEM is higher than in the case of AEM. This fact could be related to the junction potential in reference electrodes since Kingsbury et al [36] showed that perm-selectivity values for AEMs were systematically lower than those for CEM when reference electrodes with junction were used.

As for the transmission coefficient, in the case of CEM it seems to be slightly increasing with the concentration whereas for AEM it remains roughly constant. The osmotic permeability determined experimentally is the product of two parameters: mechanical permeance and salt transmission coefficient (see Eq. (3)). The lack of pronounced dependence of transmission coefficient on salt concentration is in disagreement with the classical Donnan-exclusion model. However, a qualitatively similar behaviour was also observed with Nafion 120 cation-exchange membrane [37]. 

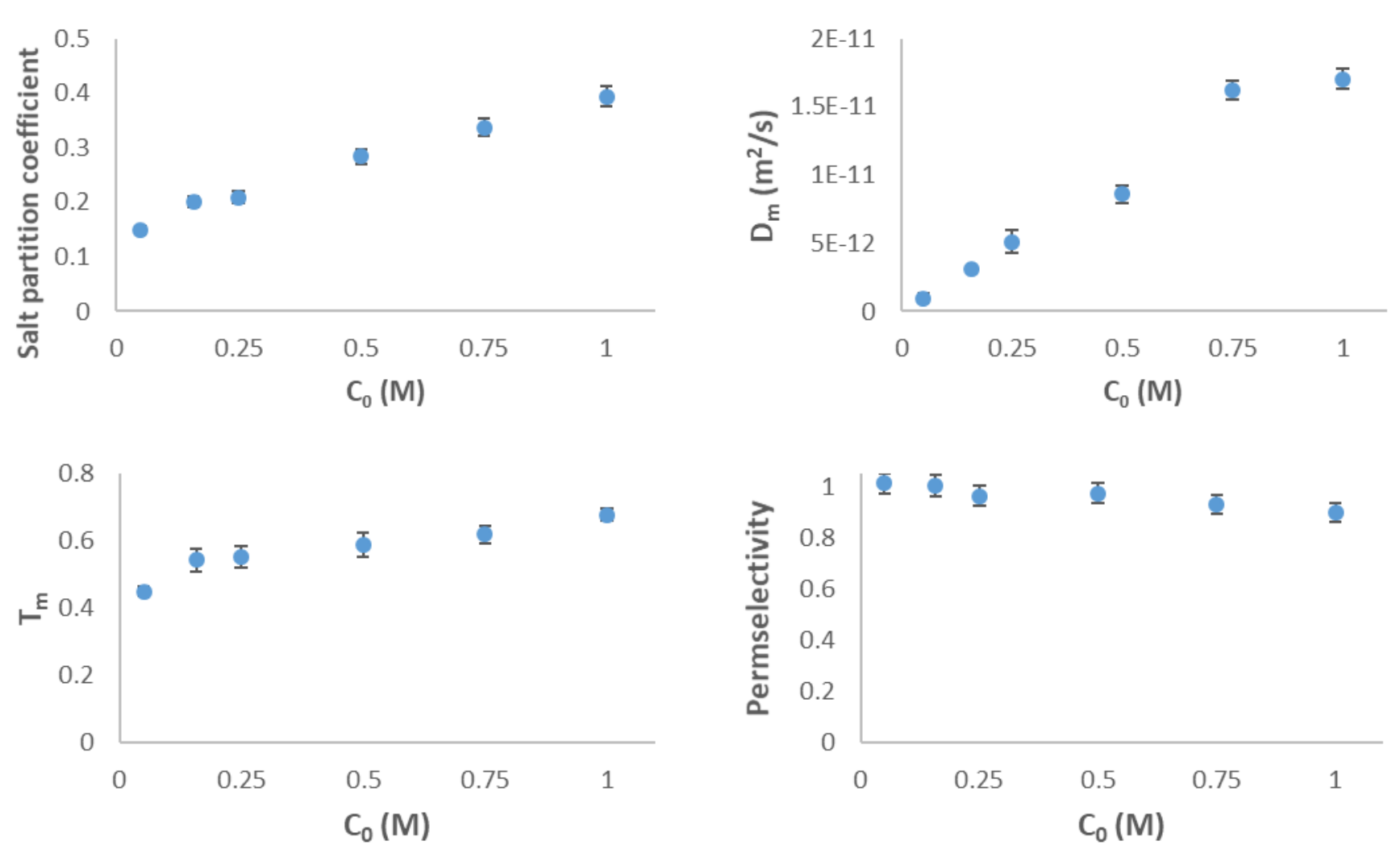

Fig.11 Properties determined for Type 10 Fujifilm CEM 

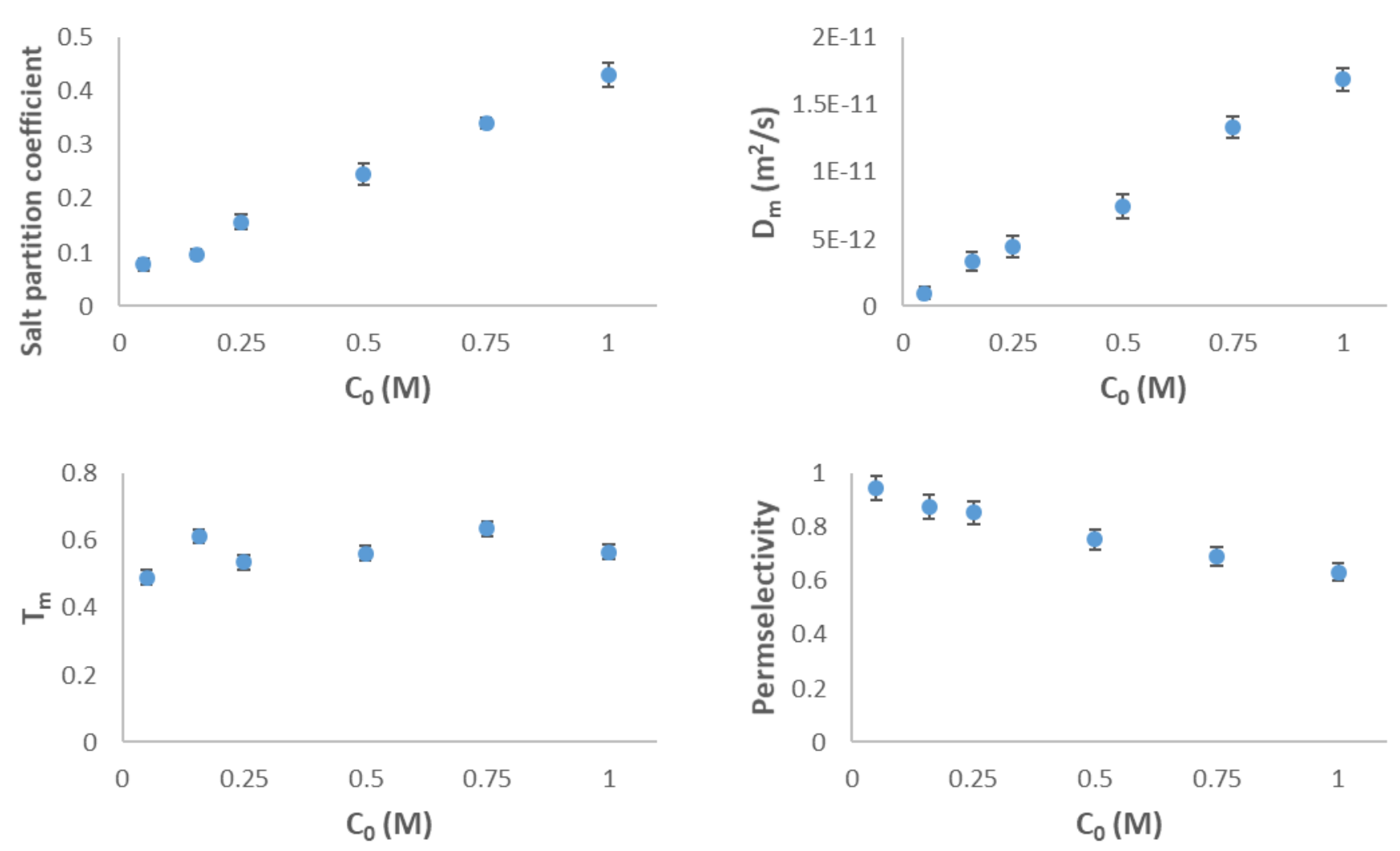

Fig.12 Properties determined for Type 10 Fujifilm AEM

\section{Conclusions}

Ion-exchange membranes are central elements of electro-membrane processes. For their optimization, it is important to have detailed information on the transport and equilibrium properties of ion-exchange membranes, in particular, separate information on the partitioning and diffusivity properties of the membrane with respect to ions.

To obtain this information, a novel approach of non-stationary diffusion (tracked via transient membrane potential) under conditions of relatively small concentration differences has been developed. It allows for a relatively simple determination of effective salt diffusion and partitioning coefficients in addition to the ion permselectivity, which is the only parameter provided by conventional stationary measurements of membrane potential.

Osmosis was included in the mathematical model developed to interpret the results. This model has been validated via comparison with (quasi)analytical solutions available in limiting cases. Osmotic permeability was determined in separate experiments. 
Systematic studies have been carried out at various $\mathrm{NaCl}$ concentrations for two different types of membrane. The new method was validated via comparison with the literature, so it can be used for systematic characterization of ion-exchange membranes.

\section{List of symbols}

$a_{0}$ : activity of base solution

$a_{i}$ : activity at the interface membrane/porous support

$a_{\text {step }}:$ activity of step solution

A: membrane mechanical permeance

$C_{0}$ : concentration of base solution

$C_{i}$ : concentration at the interface membrane/porous support

$C_{m}$ : concentration at the membrane surface

$C_{s}$ : concentration in the porous support

$C_{\text {step }}$ : concentration of step solution

$c_{X}$ : fixed-charge concentration

$d_{f}$ : glass frit diameter of the porous part

$D_{S}^{(m)}$ : effective salt diffusion coefficient in the membrane without electrostatic correction

$D_{m}$ : salt diffusion coefficient in the membrane

$D_{s}$ : salt diffusion coefficient in the porous support

$D_{s}^{(e f f)}$ : effective diffusion coefficient of salt in the frit (accounting for the porosity and tortuosity)

$D_{+}$: diffusion coefficient of cation

$D_{-}$: diffusion coefficient of anion

$F$ : Faraday constant

$H(\omega)$ : Fourier transform of unit-step function

$J_{\text {salt }}$ : salt molar flux

$J_{v}$ : osmotic flow

K: osmotic permeability

$L_{m}$ : thickness of the membrane

$L_{s}$ : thickness of the frit

$P e$ : Péclet number

r: model parameter defined by Eq. (A3)

$\mathrm{R}$ : ideal gas constant 
t: time

$t_{0}$ : characteristic relaxation time defined by Eq. (A2)

$t_{+}^{m}:$ transport number of positive ions in the membrane

$t_{+}^{b}$ : transport number of positive ions in the frit

$t_{\text {step }}$ : duration of the step $(0.001 \mathrm{~s})$

$T$ : absolute temperature

$T_{m}$ : salt transmission coefficient in membrane

$\mathrm{x}$ : longitudinal coordinate

$y$ : concentration scaled on fixed-charge concentration

$y_{0}$ : concentration of base solution scaled on fixed-charge concentration

$y_{i}$ : concentration at the interface scaled on fixed- charge concentration

$y_{s}:$ concentration of step solution scaled on fixed-charge concentration

\section{Greek letters}

$\alpha_{m}$ : chemical capacity of the membrane

$\alpha_{s}$ : chemical capacity of the frit

$\beta$ : model parameter defined by Eq. (B10)

$\delta(\omega)$ : delta function

$\theta$ : model parameter defined by Eq. (B11)

$\varphi$ : electrical potential

$\varphi_{0}:$ initial electrical potential difference

$\rho$ : model parameter defined by Eq. (A4)

$\gamma:$ salt activity coefficient in the virtual solution

$\gamma_{i}$ : salt activity coefficient at the membrane/support interface

$\gamma_{0}$ : salt activity coefficient at the initial concentration

$\gamma_{\text {step }}$ : salt activity coefficient of the non-equilibrium solution

$v$ : stoichiometric coefficient

$\Gamma_{ \pm}:$partition coefficient

$\lambda$ : parameter defined by Eq. (XX)

$\Delta \mu_{S}:$ relative salt chemical potential difference across the support

$\tau$ : dimensionless time scaled on the characteristic relaxation time defined by Eq. (A2)

$\chi$ : mechanical permeance

$\omega$ : dimensionless circular frequency 


\section{Acknowledgements}

This work has been performed within the scope of RED-Heat-to-Power project (Conversion of Low Grade Heat to Power through closed loop Reverse Electro- Dialysis) - Horizon 2020 Programme, Grant Agreement n. 640667.

\section{Supplementary information.}

\section{Model validation}

In this section, the model presented to interpret the data will be validated using different approaches. First, the PDE system resolution procedure suggested will be compared with a well-stablished model in the literature. The temporal response can be obtained through inverse Fourier transform provided that osmosis is disregarded $[27,28]$. Therefore, the PDE system will be numerically solved neglecting osmosis $(K=0)$ and the obtained result will be compared with the inverse Fourier transform solution.

Then, the concentration in the interface membrane/porous support will be determined assuming stationary conditions. The model based on the PDE resolution will be solved including osmosis and the results at very long times will be compared with the solution obtained assuming stationary conditions. The results must coincide.

\section{Model validation neglecting osmosis}

Previously, it was demonstrated that at negligible osmosis an analytical solution of Eq. (3) can be obtained via Fourier transforms. The Fourier transform of relative saltchemical-potential difference across the support, $\Delta \mu_{S}$, (scaled on the initial chemicalpotential difference) can be shown to be given by this $[27,28]$ :

$$
\Delta \mu_{S}(\omega)=\frac{H(\omega)}{\cosh (\sqrt{-i \omega})+r \cdot \sinh (\sqrt{-i \omega}) \cdot \operatorname{coth}(r \cdot \rho \cdot \sqrt{-i \omega})}
$$


where $\omega$ is the dimensionless circular frequency defined as $\omega=2 \pi f t_{0}, f$ is the dimensional frequency, $t_{0}$ is the characteristic relaxation time:

$t_{0}=\frac{\alpha_{m}}{D_{m}} \cdot L_{m}^{2}$

$r, \rho$ are model parameters defined as

$r^{2}=\frac{\alpha_{s} D_{s}}{\alpha_{m} D_{m}}$

$\rho=\frac{D_{m}}{D_{s}} \cdot \frac{L_{s}}{L_{m}}$

where $\alpha$ is the chemical capacity, $D$ is the effective diffusion coefficient and $L$ is the thickness. The indexes $m, s$ refer to the properties of membrane and porous support respectively.

$H(\omega)=\sqrt{\frac{\pi}{2}} \cdot \delta(\omega)+\frac{1}{\sqrt{2 \pi}} \cdot \frac{i}{\omega}$

is the Fourier transform of unit-step function.

The temporal response is obtained through the inverse Fourier transform:

$\Delta \mu_{s}(\tau)=\frac{1}{2} \cdot \frac{\rho}{\rho+1}+\frac{i}{2 \pi} \int_{-\infty}^{\infty} \frac{d \omega}{\omega} \cdot \frac{\exp (-i \omega \tau)}{\cosh (\sqrt{-i \omega})+r \cdot \sinh (\sqrt{-i \omega}) \cdot \operatorname{coth}(r \cdot \rho \cdot \sqrt{-i \omega})}$

where $\tau \equiv t / t_{0}$. This solution will be used below for the validation of the numerical procedure.

$\alpha_{s}$ and $D_{s}$ were obtained from glass frit characterization (see Table 1), membrane and support thicknesses are also known $\left(L_{m}=150 \mu \mathrm{m}\right.$ and $\left.L_{s}=3.7 \mathrm{~mm}\right)$, and for the validation purpose, $\alpha_{m}$ and $D_{m}$ will be set arbitrarily.

Then, the parameters $r, \rho, t_{0}$ and $\tau$ are obtained and the inverse Fourier transform integral in Eq. (11) is taken numerically, obtaining as a result the salt chemical potential difference across the support. On the other hand, the direct numerical resolution of PDE with the boundary condition described above gives the time-evolution of concentration at the interface between membrane and support, $C_{i}$. Fig.11 presents the theoretical curves obtained by both methods. Both solutions are practically identical in all the cases. 


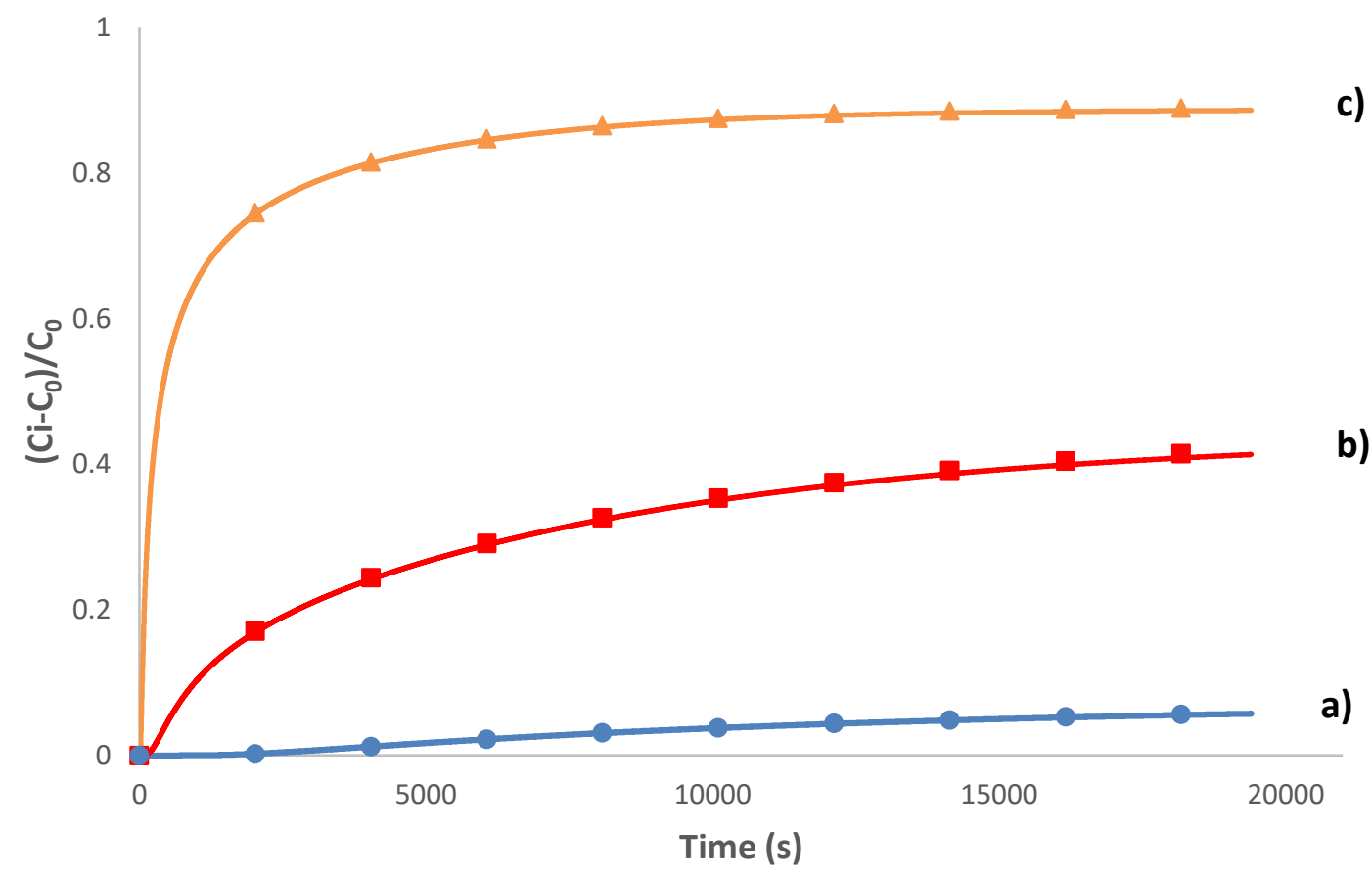

Fig.11 Concentration difference across the support scaled on the concentration step neglecting osmosis, calculated using inverse Fourier transform (dots) and direct PDE solution (lines) for: a) $\alpha_{m}=0.5 D_{m}=5 \cdot 10^{-13} m^{2} / s$, b) $\alpha_{m}=0.5 D_{m}=5 \cdot 10^{-12} m^{2} / s$ and c) $\alpha_{m}=0.5 D_{m}=5 \cdot 10^{-11} \mathrm{~m}^{2} / \mathrm{s}$

\section{Model validation accounting for osmosis}

Assuming stationary state, Eq. (3) becomes

$$
\left(-D_{m} \cdot \frac{d C_{m}}{d x}+J_{v} \cdot T_{m} \cdot C_{m}\right)=J_{S a l t}
$$

By solving Eq. (A7) in the membrane and support and using the boundary conditions of continuous concentration (at the interface) and constant salt flux (due to the steady state) we obtain

$\frac{C_{i}-C_{s t e p} \cdot \exp \left(\frac{J v \cdot T_{m}}{D_{m}} \cdot L_{m}\right)}{1-\exp \left(\frac{J_{v} \cdot T_{m}}{D_{m}} \cdot L_{m}\right)} \cdot T_{m}=\frac{C_{i}-C_{0} \cdot \exp \left(\frac{-J v}{D_{s}} \cdot L_{s}\right)}{1-\exp \left(\frac{-J_{v}}{D_{s}} \cdot L_{s}\right)}$

Now, the model based on the PDE resolution will be solved including osmosis and the results will be validated by comparing with the solution of Eq. (A8). Taking into account osmosis requires adding two parameters: osmotic permeability, $K$, where the values determined experimentally will be used (see Table 2 ), and salt transmission coefficient, $T_{m}$, which will be set arbitrarily at 0.5 just for validation purposes. 


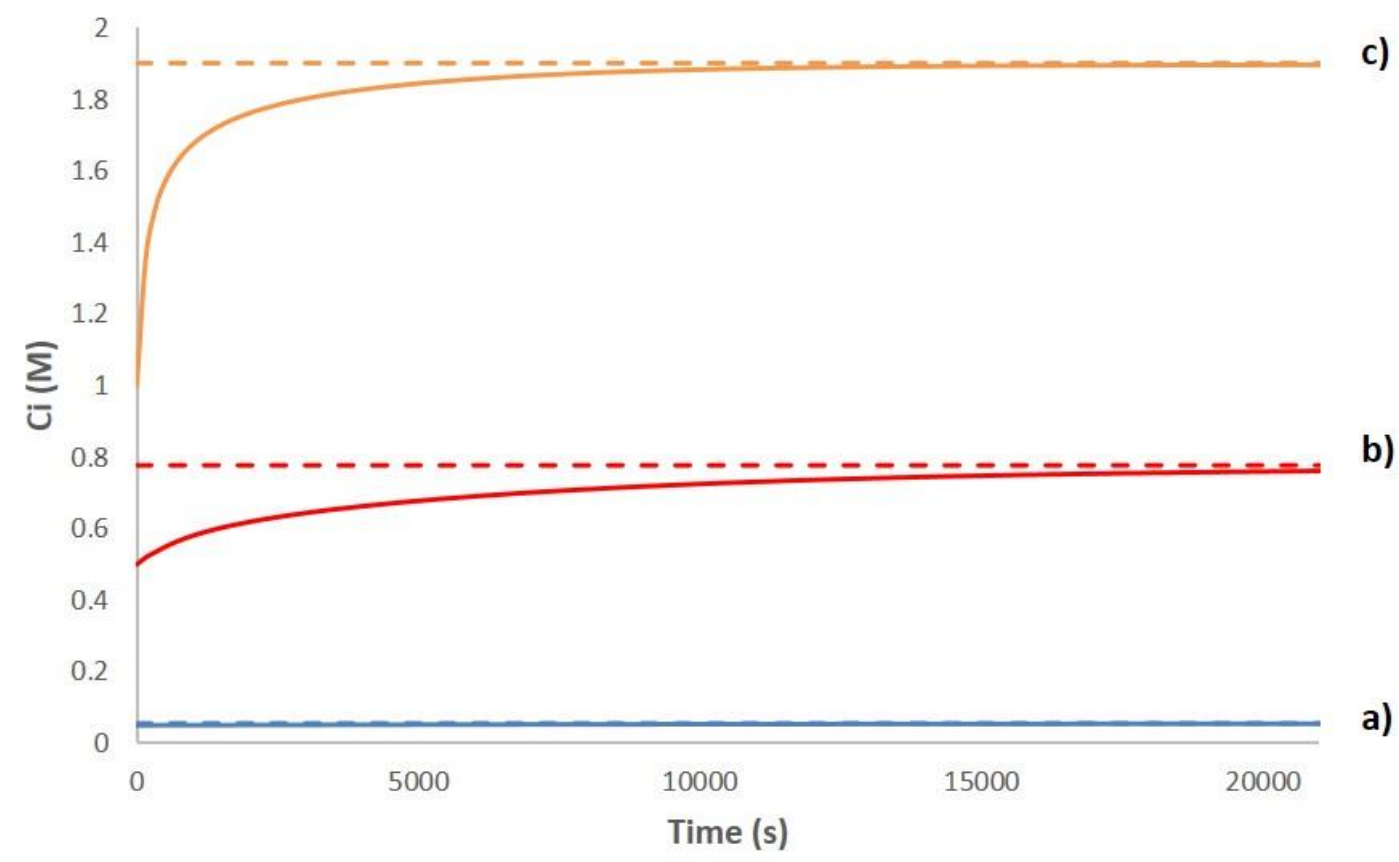

Fig.12 Concentration at the interface between membrane and porous support obtained from PDE system resolution (solid line) and stationary value calculated with

Eq. (18) (dotted line) for: a) $\boldsymbol{\alpha}_{m}=\mathbf{0 . 5}, \boldsymbol{D}_{m}=\mathbf{5} \cdot \mathbf{1 0}^{-13} \mathrm{~m}^{2} / \mathrm{s}$, b) $\boldsymbol{\alpha}_{m}=\mathbf{0 . 5}, \boldsymbol{D}_{m}=\mathbf{5}$. $10^{-12} \mathrm{~m}^{2} / \mathrm{s}$ and c) $\alpha_{m}=0.5 D_{m}=5 \cdot 10^{-11} \mathrm{~m}^{2} / \mathrm{s}$

Fig.12 shows the comparison between the PDE system solution and the value at steady state calculated with Eq. (A8). In all cases, the PDE solution tends to the stationary values.

\section{Osmosis contribution}

In this section we evaluate the osmosis contribution by comparing the theoretical curves calculated taking into account osmosis and disregarding it. The parameters used in these calculations were obtained from the fitting of experimental data to the model (see above).

Moreover, standard Donnan model will be used under stationary conditions to take into account the concentration dependence of membrane transport properties. This will help us understand some unexpected experimental behavior discussed below.

Standard Donnan model assumes a Donnan-equilibrium at the membrane/solution interfaces [38]. Within the membrane there are fixed charge groups. Ion distribution 
coefficients are related to each other by the condition of local electric neutrality. All the ion distribution coefficients can be expressed through the Donnan potential.

Stationary transport equations in the membrane and in the porous support can be written down in this way:

$$
\begin{aligned}
& J_{\text {salt }}=-P_{s}(c) \cdot \frac{\partial c_{m}}{\partial x}+c_{m} \cdot \mathrm{T}_{s}(c) \cdot J_{v} \\
& D_{s}^{(e f f)} \cdot \frac{d c}{d x}=c \cdot j_{v}-j_{s} \\
& J_{s a l t}=-D_{s}^{e f f} \cdot \frac{\partial c_{s}}{\partial x}+J_{v} \cdot c_{s}
\end{aligned}
$$

Where $J_{\text {salt }}$ is the salt molar flux, $c_{m}, c_{s}$ are the concentration in the membrane and in the porous support respectively, $x$ is the longitudinal coordinate, $P_{S}$ is membrane permeability, $T_{S}$ is the salt transmission coefficient, $D_{S}^{\text {eff }}$ is the effective diffusion coefficient of salt in the porous support (accounting for porosity and tortuosity) and $J_{v}$ is the osmotic flow.

Within the scope of Donnan model ((1:1) salt, ideal solution) [38]:

$$
\begin{aligned}
& T_{s}(c)=\frac{2 \cdot c / c_{X}}{\sqrt{1+\left(2 \cdot c / c_{X}\right)^{2}}+a} \equiv \frac{y}{\sqrt{1+y^{2}}+a} \\
& P_{s}(c)=\frac{D_{s}^{(m)} \cdot 2 \cdot c / c_{X}}{\sqrt{1+\left(2 \cdot c / c_{X}\right)^{2}}+a} \equiv \frac{D_{s}^{(m)} \cdot y}{\sqrt{1+y^{2}}+a}
\end{aligned}
$$

With the following notations:

$\lambda \equiv \frac{D_{+}-D_{-}}{D_{+}+D_{-}}$

$y \equiv 2 \cdot c / c_{X}$

$D_{S}^{(m)}$ is the effective salt diffusion coefficient in the membrane without electrostatic correction and $c_{X}$ is the fixed-charge concentration.

The boundary conditions of continuous concentration and constant salt flux apply. The equation in the membrane is a first-order equation with separable variables that can be solved in quadratures, obtaining as a result a transcendental equation for the dimensionless interface concentration:

$$
\int_{y_{i}^{2}}^{y_{s}^{2}} \frac{d \xi}{\lambda \cdot \xi+\frac{y_{i} \cdot e^{-P e}-y_{0}}{1-e^{-P e}} \cdot(\sqrt{1+\xi}+\lambda)}=2 P e \cdot \beta
$$


$P e=\theta \cdot\left[\left(y_{s}-y_{i}\right)-\alpha \cdot\left(\sqrt{y_{s}^{2}+1}-\sqrt{y_{i}^{2}+1}-a \cdot \ln \left(\frac{a+\sqrt{y_{s}^{2}+1}}{a+\sqrt{y_{i}^{2}+1}}\right)\right)\right]$

$\beta \equiv \frac{L_{m}}{D_{s}^{(m)}} \cdot \frac{D_{s}^{(e f f)}}{L_{s}}$

$\theta \equiv \frac{R T A L_{s} c_{X}}{D_{s}^{(e f f)}}$

$A$ is the membrane mechanical permeance.

(a)

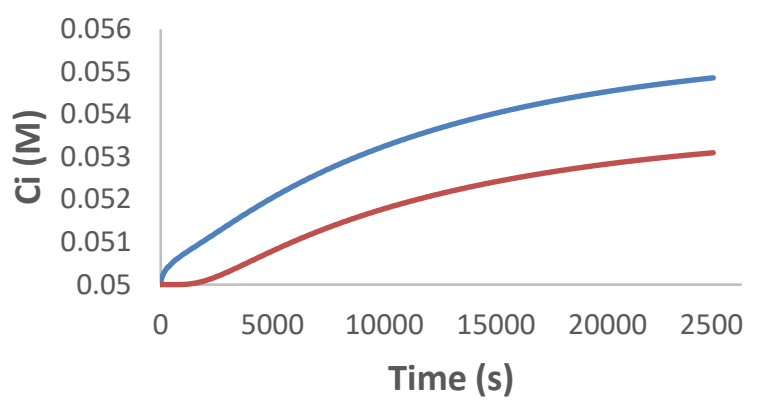

Osmosis No osmosis (b)

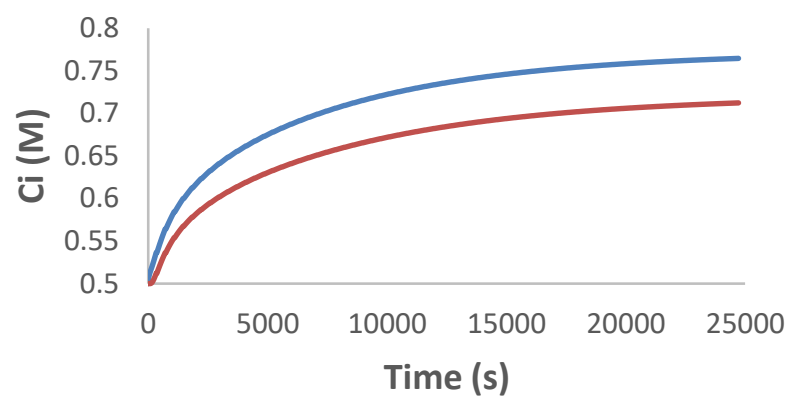

Osmosis $\longrightarrow$ No osmosis

(c)

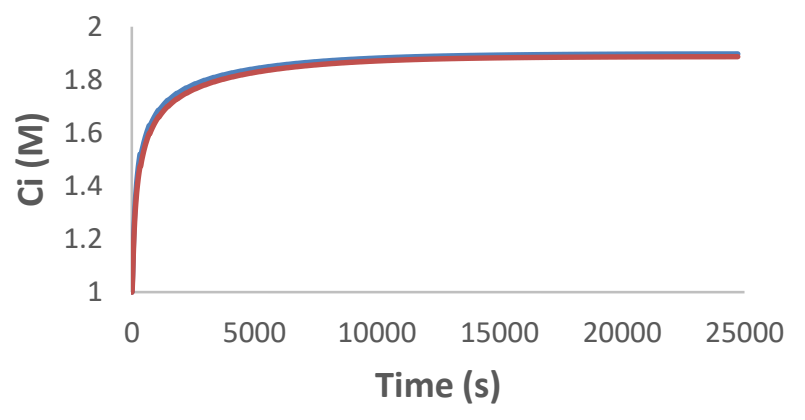

- Osmosis - No osmosis

Fig.13 Osmosis effect on the time evolution of salt concentration at the interface between membrane and porous support for: a) $\alpha_{m}=\mathbf{0 . 1 4}, D_{m}=\mathbf{9 . 7 4} \cdot 10^{-13} \mathrm{~m}^{2} / \mathrm{s}$, $T_{m}=0.45$ b) $\alpha_{m}=0.39, D_{m}=7.80 \cdot 10^{-12} m^{2} / s, T_{m}=0.59$ and c) $\alpha_{m}=0.60, D_{m}=1.71$. $10^{-11} \mathrm{~m}^{2} / \mathrm{s}, T_{m}=0.68$

As can be seen from Fig.13, there is a considerable impact, especially at shorter times, and in more dilute solutions whereas it becomes less pronounced when the concentration increases. Due to osmosis the relaxation of the signal becomes slower, 
which is logical since the osmotic flow goes in the opposite direction to the diffusion. The decrease of relative contribution of osmosis with increasing concentration has been less expected because usually osmosis gets more important with increasing concentration. The opposite trend in our case is primarily due to the strong increase of diffusion permeability of ion-exchange membrane with concentration. The bi-layer structure of our system also plays a role. This is illustrated by Fig.14 that shows relative difference between the stationary (very long time) values of interface concentration calculated by accounting for osmosis and disregarding it within the scope of classical Donnan model.

Although Donnan model is not quantitatively applicable, it captures the strong dependence of membrane diffusion permeability on salt concentration.

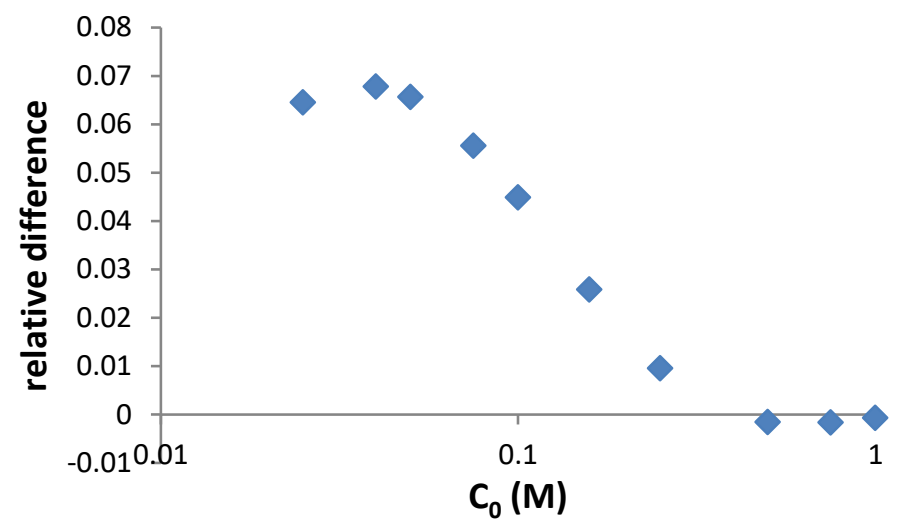

Fig.14 Relative difference between interface concentration calculated considering osmosis and neglecting it $\left(c_{X}=2000 \mathrm{~mol} / \mathrm{m}^{3}, D_{s}^{(m)}=3.41 \cdot 10^{-11} \mathrm{~m}^{2} / \mathrm{s}\right)$

One can see that within a broad concentration range the relative contribution of osmosis, indeed, decreases with increasing concentration. Moreover, at a concentration around $\mathrm{C}_{0}=1 \mathrm{M}$ this contribution changes sign. This may explain the very weak impact of osmosis predicted at $C_{0}=1 M$.

\section{References}

[1] T. Luo, S. Abdu, M. Wessling, Selectivity of Ion Exchange Membranes: A Review, (2018). doi:10.1016/j.memsci.2018.03.051.

[2] M. Micari, M. Bevacqua, A. Cipollina, A. Tamburini, W. Van Baak, T. Putts, G. Micale, E ff ect of di ff erent aqueous solutions of pure salts and salt mixtures in reverse electrodialysis systems for closed-loop applications, J. Memb. Sci. 551 (2018) 315-325. doi:10.1016/j.memsci.2018.01.036.

[3] A. Tamburini, M. Tedesco, A. Cipollina, G. Micale, M. Ciofalo, M. Papapetrou, W. Van Baak, A. Piacentino, Reverse electrodialysis heat engine for sustainable power production, Appl. Energy. 206 (2017) 1334-1353. doi:10.1016/j.apenergy.2017.10.008.

[4] M. Reig, X. Vecino, C. Valderrama, O. Gibert, J.L. Cortina, Separation and Puri fi cation 
Technology Application of selectrodialysis for the removal of As from metallurgical process waters: Recovery of Cu and Zn, Sep. Purif. Technol. 195 (2018) 404-412. doi:10.1016/j.seppur.2017.12.040.

[5] M. Reig, S. Casas, C. Valderrama, O. Gibert, J.L. Cortina, Integration of monopolar and bipolar electrodialysis for valorization of seawater reverse osmosis desalination brines : Production of strong acid and base, DES. 398 (2016) 87-97. doi:10.1016/j.desal.2016.07.024.

[6] T. Rijnaarts, N.T. Shenkute, A. Wood, W.M. De Vos, K. Nijmeijer, Divalent Cation Removal by Donnan Dialysis for Improved Reverse Electrodialysis, (2018). doi:10.1021/acssuschemeng.8b00879.

[7] C. Agarwal, R.W. Cattrall, S.D. Kolev, Donnan dialysis based separation of gold ( III ) from electronic waste solutions using an anion exchange pore- fi lled membrane, J. Memb. Sci. 514 (2016) 210-216. doi:10.1016/j.memsci.2016.04.033.

[8] A.N. Naik, C. Agarwal, S. Chaudhury, A. Goswami, Non-stationary radiotracer method for diffusion coefficients of Cs , Ba , Eu tracers in Nafion-117 membrane, 6395 (2016). doi:10.1080/01496395.2016.1256324.

[9] R. Bernstein, Y. Kaufman, V. Freger, Membrane Characterization, Encycl. Membr. Sci. Technol. (2013) 41. doi:10.1002/elan.

[10] G.M. Geise, B.D. Freeman, D.R. Paul, Sodium chloride diffusion in sulfonated polymers for membrane applications, J. Memb. Sci. 427 (2013) 186-196. doi:10.1016/j.memsci.2012.09.029.

[11] V. Freger, A. Ben-David, Use of attenuated total reflection infrared spectroscopy for analysis of partitioning of solutes between thin films and solution, Anal. Chem. 77 (2005) 6019-6025. doi:10.1021/ac050689w.

[12] A. Ben-David, Y. Oren, V. Freger, Thermodynamic factors in partitioning and rejection of organic compounds by polyamide composite membranes, Environ. Sci. Technol. 40 (2006) 7023-7028. doi:10.1021/es0609912.

[13] A. Ben-David, S. Bason, J. Jopp, Y. Oren, V. Freger, Partitioning of organic solutes between water and polyamide layer of RO and NF membranes: Correlation to rejection, J. Memb. Sci. 281 (2006) 480-490. doi:10.1016/j.memsci.2006.04.017.

[14] A. Ghoufi, E. Dražević, A. Szymczyk, Interactions of Organics within Hydrated Selective Layer of Reverse Osmosis Desalination Membrane: A Combined Experimental and Computational Study, Environ. Sci. Technol. 51 (2017) 2714-2719. doi:10.1021/acs.est.6b05153.

[15] X. Zhang, D.G. Cahill, O. Coronell, B.J. Mariñas, Partitioning of salt ions in FT30 reverse osmosis membranes, Appl. Phys. Lett. 91 (2007). doi:10.1063/1.2802562.

[16] B. Mi, B.J. Mariñas, D.G. Cahill, RBS characterization of arsenic(III) partitioning from aqueous phase into the active layers of thin-film composite NF/RO membranes, Environ. Sci. Technol. 41 (2007) 3290-3295. doi:10.1021/es062292v.

[17] J. Wang, R.S. Kingsbury, L.A. Perry, O. Coronell, Partitioning of Alkali Metal Salts and Boric Acid from Aqueous Phase into the Polyamide Active Layers of Reverse Osmosis Membranes, Environ. Sci. Technol. 51 (2017) 2295-2303. doi:10.1021/acs.est.6b04323.

[18] I. Nicotera, C. Simari, L.G. Boutsika, L. Coppola, K. Spyrou, A. Enotiadis, NMR investigation on nanocomposite membranes based on organosilica layered materials bearing different functional groups for PEMFCs, Int. J. Hydrogen Energy. 42 (2017) 27940-27949. 
doi:10.1016/j.ijhydene.2017.05.014.

[19] R. Mueller, V. Hariharan, C. Zhang, R. Lively, S. Vasenkov, Relationship between mixed and pure gas self-diffusion for ethane and ethene in ZIF-8/6FDA-DAM mixed-matrix membrane by pulsed field gradient NMR, J. Memb. Sci. 499 (2016) 12-19. doi:10.1016/j.memsci.2015.10.036.

[20] E. Drazevic, S. Bason, K. Kosutic, V. Freger, Enhanced partitioning and transport of phenolic micropollutants within polyamide composite membranes, Environ. Sci. Technol. 46 (2012) 3377-3383. doi:10.1021/es204188j.

[21] J. Kamcev, D.R. Paul, G.S. Manning, B.D. Freeman, Accounting for frame of reference and thermodynamic non-idealities when calculating salt diffusion coefficients in ion exchange membranes, J. Memb. Sci. 537 (2017) 396-406. doi:10.1016/j.memsci.2017.05.034.

[22] A. Cañas, M.J. Ariza, J. Benavente, Characterization of active and porous sublayers of a composite reverse osmosis membrane by impedance spectroscopy, streaming and membrane potentials, salt diffusion and X-ray photoelectron spectroscopy measurements, 183 (2001) 135-146.

[23] A. Goswami, A. Acharya, A.K. Pandey, Membrane, (2001) 9196-9201. doi:10.1021/jp010529y.

[24] V. Compan, M.L. Lopez, T.S. Soerensen, J. Garrido, Transport Numbers in the Surface Layers of Asymmetric Membranes from Initial Time Measurements, J. Phys. Chem. 98 (1994) 9013-9021. doi:10.1021/j100087a033.

[25] V. Compañ, T.S. Sorensen, S.R. Rivera, Comparison of Initial Time and Stationary State Measurements of the Emf of Concentration Cells Using Phenolsulfonic Acid Membrane Separators, (1995) 12553-12558.

[26] V. Compañ, T.S. Sorensen, A. Andrio, D. Abajo, Transport numbers from initial time and stationary state measurements of EMF in non-ionic polysulphonic membranes, 8 (1997) 7-10.

[27] A. Yaroshchuk, Y. Boiko, A. Makovetskiy, Electrochemical perm-selectivity of active layers and diffusion permeability of supports of an asymmetric and a composite NF membrane studied by concentration-step method, Desalination. 245 (2009) 374-387. doi:10.1016/j.desal.2009.02.001.

[28] A.E. Yaroshchuk, A.L. Makovetskiy, Y.P. Boiko, E.W. Galinker, Non-steady-state membrane potential: Theory and measurements by a novel technique to determine the ion transport numbers in active layers of nanofiltration membranes, J. Memb. Sci. 172 (2000) 203-221.

[29] A. Yaroshchuk, X. Martínez-Lladó, L. Llenas, M. Rovira, J. de Pablo, Solution-diffusion-film model for the description of pressure-driven trans-membrane transfer of electrolyte mixtures: One dominant salt and trace ions, J. Memb. Sci. 368 (2011) 192-201. doi:10.1016/j.memsci.2010.11.037.

[30] A. Yaroshchuk, O. Zhukova, M. Ulbricht, V. Ribitsch, Electrochemical and Other Transport Properties of Nanoporous Track-Etched Membranes Studied by the Current Switch-Off Technique, (2005) 6872-6882. doi:10.1021/la050499g.

[31] A.E. Yaroshchuk, V. Ribitsch, The uses of non-steady-state membrane characterisation techniques for the study of transport properties of active layers of nanofiltration membranes : theory with experimental examples, 80 (2000) 203-214.

[32] W.J. Hamer, Y.-C. Wu, Osmotic coefficients and Mean Activity Coefficients of Uni- 
univalent Electrolytes in Water at $25^{\circ} \mathrm{C}$, J. Phys. Chem. Ref. Data. 1 (1972) 1047-1100.

[33] G.M. Geise, H.J. Cassady, D.R. Paul, E. Logan, M.A. Hickner, Specific ion effects on membrane potential and the permselectivity of ion exchange membranes, Phys. Chem. Chem. Phys. 16 (2014) 21673-21681. doi:10.1039/C4CP03076A.

[34] A. Narębska, S. Koter, W. Kujawski, lons and water transport across charged nafion membranes. Irreversible thermodynamics approach, Desalination. 51 (1984) 3-17. doi:10.1111/j.1467-9353.2006.00281.x.

[35] E.H. Cwirko, R.G. Carbonell, Ionic equilibria in ion-exchange membranes: a comparison of pore model predictions with experimental results, 67 (1992) 211-226. doi:10.1016/0376-7388(92)80026-g.

[36] R.S. Kingsbury, S. Flotron, S. Zhu, D.F. Call, O. Coronell, Junction Potentials Bias Measurements of Ion Exchange Membrane Permselectivity, Environ. Sci. Technol. 52 (2018) 4929-4936. doi:10.1021/acs.est.7b05317.

[37] W. Kujawski, A. Narebska, Transport of electrolytes across charged membranes. Part IV. Frictional interactions of the neutral and alkaline permeants and the permeability/reflection phenomena*, 56 (1991) 99-112.

[38] A.E. Yaroshchuk, Negative rejection of ions in pressure-driven membrane processes, Adv. Colloid Interface Sci. 139 (2008) 150-173. doi:10.1016/j.cis.2008.01.004. 